\title{
LA PERSPECTIVA DE GÉNERO EN LA CARRERA DE GEOGRAFIA DE LA UNNE. UN ESBOZO DE COMPARACIÓN CON LA INCORPORACIÓN DEL GÉNERO A LA CIENCIA GEOGRAFICA.
}

\section{GENDER IN THE GEOGRAPHY OF RACE UNNE. AN OUTLINE OF COMPARISON WITH THE INTRODUCTION OF GEOGRAPHICAL SCIENCE GENDER}

\author{
Mgtr. Vilma Lilian Falcón \\ Profesora Adjunta \\ Departamento de Geografía -Facultad de Humanidades
}

vfalcon@hum.unne.edu.ar

\section{Resumen}

El propósito de esta presentación es hacer una revisión de la incorporación de la teoría de género a la disciplina geográfica en general y de un modo más específico a la Geografía que se enseña e investiga a través de los planes de estudio del profesorado y licenciatura en Geografía de la Facultad de Humanidades de la Universidad Nacional del Nordeste (UNNE).

Una de las primeras reflexiones que considero relevante destacar es que la fuerte influencia de la corriente neopositivista en el diseño curricular de la carrera en geografía ha marcado, desde sus inicios a fines de la década del 50, una línea muy difícil de flexionar a tal punto que en los momentos actuales solo una ínfima parte de los programas curriculares del profesorado y licenciatura en Geografía exponen tímidamente temas relacionados con las "cuestiones de género".

En este trabajo se expone brevemente el proceso que siguió la incorporación de la perspectiva de género en la ciencia geográfica y en un segundo momento se realiza un estado de situación de la Geografía académica que se ofrece en la región a través de nuestro profesorado y licenciatura en Geografía, destacando especialmente el período en que se llevó a cabo el proceso de cambio curricular realizado en el marco de la reforma educativa a fines de la década de los '90 y la consecuente propuesta del Plan de Estudio 2000 actualmente vigente.

Como resultado de ese análisis surgen algunas consideraciones que destacan los avances logrados hasta el momento y fundamentalmente las cuestiones que aún están pendientes lograr para que el género comience a ocupar el nivel de categoría explicativa en nuestra propuesta curricular.

Palabras Claves: Perspectiva de género - Geografía de Género -Ciencia Geográfica

\section{Sumary}

This proposal consists of reviewing the incorporation of the gender theory in the discipline of general Geography and more specifically in the Geography taught and researche dacross the curricula for the Geography teacher training and bachelor's degree at the Faculty of Humanities of the Northeastern University (UNNE).

One of the first thoughts that I consider relevant to emphasize is that the strong influence of the neopositivist school of thought over the development of the curriculum for the geography teacher career has marked, from its beginnings in the late 50 s, a very hard line, to the point that at present only a tiny part of the study programs for the teacher training and bachelor's degree in Geography slightly presents topics related to "gender issues".

This paper briefly exposes first the process that followed the incorporation of the gender perspective in the geographical science and secondly it carries out a situation state of the academic Geography offered in the region by our faculty. It is especially highlighted the period in which the process of curriculum change was accomplished, in the context of the educational reform in the late

Publicado en formato digital: Mgtr. Vilma Lilian Falcón. LA PERSPECTIVA DE GÉNERO EN LA CARRERA DE GEOGRAFIA DE LA UNNE. UN ESBOZO DE COMPARACIÓN CON LA INCORPORACIÓN DEL GÉNERO A LA CIENCIA GEOGRAFICA. Revista Geográfica Digital. IGUNNE. Facultad de Humanidades. UNNE. Año 10. № 19. Enero - Junio 2013. ISSN 1668-5180 Resistencia, Chaco. En:

http://hum.unne.edu.ar/revistas/geoweb/default.htm 
'90s and the subsequent proposal for the Curriculum 2000, currently in effect. As a result of this analysis raise some considerations that point out the progress made so far, and mainly the issues that are still outstanding for the genre to start occupying the level of explanatory category in our proposed curriculum.

Keywords: Gender Perspective - Gender Geography- Geographical Science 
Revista Geográfica Digital. IGUNNE. Facultad de Humanidades. UNNE. Año 10. № 19. Enero - Junio 2013. ISSN 1668-5180 Resistencia, Chaco

\section{Introducción}

La propuesta de este trabajo consiste en hacer una revisión de la incorporación de la teoría de género a la disciplina geográfica en general y de un modo más específico a la Geografía que se enseña e investiga a través de los planes de estudio del profesorado y licenciatura en Geografía de la Facultad de Humanidades, ámbito en el cual me desempeño profesionalmente.

El objetivo central de este trabajo es la búsqueda de respuestas posibles al motivo por el cual los actuales planes de estudio de las carreras en Geografía, que se dictan en nuestra Facultad de Humanidades, no han incorporado la perspectiva de género en sus diseños curriculares y, por otra parte, se pretende destacar el modo en que algunas "cuestiones de género" (1) han ido permeando ciertas unidades temáticas en diferentes cátedras correspondientes al Plan de Estudio vigente.

Una reflexión que actúa como hipótesis es que, desde sus inicios a fines de la década del '50, el diseño curricular de la carrera en Geografía se vio fuertemente influenciado por la corriente neopositivista, marcando una línea muy difícil de flexionar a tal punto que en el proceso de desarrollo que siguió la carrera, los últimos Planes de Estudio no presentaron cambios estructurales en cuanto a sus propuestas curriculares.

En general, la ciencia geográfica fue incorporando la perspectiva de género de un modo paulatino pero a la vez progresivo; lo promisorio de ese proceso es que se fue pasando del análisis simple de reconocer patrones diferenciales de las mujeres en relación con el espacio hasta el momento actual en que la perspectiva de género se está constituyendo en referencia fundamental en los debates teóricos, epistemológicos y metodológicos de la Geografía Humana (Cfr. Sabaté Martinez, Rodriguez Moya y Díaz Muñoz, 1995:23). Este proceso de debates teóricosepistemológicos es relevante en el desarrollo de cualquier ciencia porque a la luz de los enfoques teóricos se tiene la posibilidad de ver y analizar la realidad del mundo y de los Sujetos que en él vivimos desde otras perspectivas y esto no es más que lo que Celia Amorós (2) nos recuerda respecto del fin de toda teoría: "posibilitar una nueva visión, una nueva interpretación de la realidad". Entiendo que la ausencia de este proceso de discusiones teóricas que conllevan a reconceptualizaciones y reconsideraciones de categorías de análisis fue uno de los obstáculos fundamentales que tuvo nuestra ciencia geográfica, enseñada e investigada en la región Nordeste.

Hacia la revisión de estas dos cuestiones centrales se orienta este trabajo y para ello expondré brevemente, en un primer momento, el proceso que siguió la incorporación de la perspectiva de género en la ciencia geográfica, tomando como fuente principal lo que considero un verdadero manual que ofrece un gran aporte a esta temática: Mujeres, Espacio y Sociedad: hacia una Geografía de Género (3) así como los aportes de otros autores. En un segundo momento intentaré esbozar un estado de situación de la Geografía académica que se ofrece en la región a través de nuestro profesorado y licenciatura en Geografía destacando especialmente el período en que se llevó a cabo el proceso de cambio curricular realizado en el marco de la reforma educativa a fines de la década de los '90 (4) y la consecuente propuesta del Plan de Estudio 2000 actualmente vigente. Como producto de este análisis se realizan algunas consideraciones que destacan los avances logrados hasta el momento y fundamentalmente las cuestiones que aún están pendientes lograr para que el género comience a ocupar el nivel de categoría explicativa en nuestra propuesta curricular.

\section{Breve repaso de la incorporación de la perspectiva de género a la Geografía}

\section{Feminismo, Género y Geografía}

Cuando se trata de buscar los orígenes de la geografía de género numerosos autores coinciden al señalar la necesaria referencia al Feminismo como un movimiento social y político pero que, al mismo tiempo es considerado una concepción teórica y una práctica de interpretación (Cfr. Veleda Da Silva y Lan, 2007:102). En este sentido, Femenías, M.(2005-2006:46) (5), sostiene que este movimiento emerge en al menos dos vertientes: "una potente resignificación de los conceptos y un conjunto de prácticas novedosas y efectivas", aspectos que la autora sostiene van de la mano ya

Publicado en formato digital: Mgtr. Vilma Lilian Falcón. LA PERSPECTIVA DE GÉNERO EN LA CARRERA DE GEOGRAFIA DE LA UNNE. UN ESBOZO DE COMPARACIÓN CON LA INCORPORACIÓN DEL GÉNERO A LA CIENCIA GEOGRAFICA. Revista Geográfica Digital. IGUNNE. Facultad de Humanidades. UNNE. Año 10. № 19. Enero - Junio 2013. ISSN 1668-5180 Resistencia, Chaco. En:

http://hum.unne.edu.ar/revistas/geoweb/default.htm 
Revista Geográfica Digital. IGUNNE. Facultad de Humanidades. UNNE. Año 10. № 19. Enero - Junio 2013. ISSN 1668-5180 Resistencia, Chaco

que su diferenciación responde solo al análisis. Si consideramos el primer aspecto, lo que propone el feminismo como movimiento social es un cambio en la estructura científica de las ciencias sociales, siendo su objetivo primordial el introducir una nueva perspectiva -la del género- en la construcción teórica de las ciencias sociales.

Como lo señala Sabaté Martinez et al. (1995:29), tener en cuenta el aporte del feminismo a otras ciencias sociales como la historia, la sociología, la economía o la antropología, es fundamental al considerar el desarrollo de la geografía del género. A modo de ejemplo, señalan las autoras, en la economía y la sociología, el feminismo incorpora la discusión sobre los conceptos de producción y reproducción, la valoración del trabajo doméstico y la redefinición de conceptos básicos en el estudio del mercado de trabajo; en la antropología (6), las críticas al etnocentrismo de la investigación occidental, el estudio de la familia y las relaciones de parentesco, y el análisis de la construcción cultural de género; y en la Historia, incorpora el estudio de la vida cotidiana en el pasado y el reconocimiento del papel de la mujer en la evolución de la humanidad.

Si se afirma que en el desarrollo de toda ciencia existen elementos externos e internos que influyen en su desarrollo, para el caso de la ciencia geográfica, el feminismo formaría parte de los elementos externos que ayudan a entender el contexto en el cual nace y se desarrolla la Geografía del Género. Al mismo tiempo, otros elementos como la evolución de la situación de las mujeres en la sociedad y las aportaciones del resto de las ciencias sociales conforman ese marco exterior; pero también se debe tener en cuenta los cambios habidos en la estructura teórica y metodológica de la ciencia geográfica y el operar de la comunidad científica que es quien refleja los prejuicios e intereses y traduce la influencia del contexto social al interior de la propia ciencia. (Cfr. Sabaté Martinez et al.1995:24).

\section{Las corrientes teóricas y la conformación de la Geografía del género (7)}

En las décadas de los '60 y '70, la ciencia geográfica todavía mostraba un escaso interés por los planteamientos teóricos de la perspectiva de género y ello estaba dado por la fuerte influencia que ejercían las corrientes teóricas y metodológicas imperantes en ese momento en nuestra ciencia. La Geografía neopositivista, con su interés centrado en los procesos espaciales abstractos, la búsqueda de regularidad en las distribuciones espaciales, claramente objetiva y neutral en los procedimientos de investigación, tendía a excluir de su campo de estudio temas relativos al cambio social y al papel activo de las ciencias humanas entre las que se encontraba la perspectiva feminista. Solo cuando la geografía de la percepción abre las primeras fisuras en el paradigma positivista, comienzan a desarrollarse trabajos interesados por conocer el comportamiento espacial específico de las mujeres y las construcciones mentales del entorno.

A mediados de los '70, en el mundo anglosajón, surge la llamada Geografía del Bienestar, la que constituyó un marco más abierto a la consideración de temas relacionados con el género, aunque sin plantear cambios de consideración en la estructura teórica y metodológica. Es el marco que propició el surgimiento de la Geografía de las mujeres, orientada a no "excluir a la mitad de los humanos de la Geografía humana" (Janice Monk y Susan Hanson, 1982)(8). Para esta perspectiva, la desigualdad de la mujer se deriva de las restricciones espacio-temporales que le impone la división de roles según género establecidas en nuestra sociedad; ello llevó a estudiar cuestiones como la movilidad espacial, el uso y la percepción del Espacio, el acceso a los servicios, entre otros cuestionamientos que pretendían poner de relieve las limitaciones sociales y espaciales por las que atravesaban las mujeres.

Los enfoques post o antipositivistas que se extienden en el ámbito de la Geografía desde los años '70 fueron los facilitadores de la incorporación de la perspectiva de género a la investigación social; estos enfoques constituyeron un profunda revolución epistemológica, teórica y metodológica en las ciencias sociales. Aún cuando procedían de filosofías humanistas o marxistas produjeron importantes aportes a la Geografía , como por ejemplo: reconsiderar la escala de análisis en los estudios, llegando a la experiencia de la vida cotidiana; dando lugar al geógrafo de comprometerse con el cambio de las situaciones que estudia y finalmente en el paso de la mera descripción o

Publicado en formato digital: Mgtr. Vilma Lilian Falcón. LA PERSPECTIVA DE GÉNERO EN LA CARRERA DE GEOGRAFIA DE LA UNNE. UN ESBOZO DE COMPARACIÓN CON LA INCORPORACIÓN DEL GÉNERO A LA CIENCIA GEOGRAFICA. Revista Geográfica Digital. IGUNNE. Facultad de Humanidades. UNNE. Año 10. № 19. Enero - Junio 2013. ISSN 1668-5180 Resistencia, Chaco. En:

http://hum.unne.edu.ar/revistas/geoweb/default.htm 
Revista Geográfica Digital. IGUNNE. Facultad de Humanidades. UNNE. Año 10. № 19. Enero - Junio 2013.

ISSN 1668-5180 Resistencia, Chaco

explicación a la interpretación de los fenómenos geográficos a partir de procesos sociales globales o de las motivaciones y experiencias personales. Todo ello influyó también en la adopción de nuevas estrategias metodológicas pasando de lo estrictamente cuantificable a métodos cualitativos que implican una actitud más subjetiva del investigador.

La Geografía Radical Marxista, surge como otra gran alternativa epistemológica al positivismo y manifiesta rasgos comunes con la perspectiva de género ya que ambas abogan por una participación del científico en los movimientos a favor de la justicia social y en defensa de los grupos marginados, atribuyendo a la ciencia un compromiso político. El marco teórico marxista y sus categorías de análisis brindaron, a la Geografía del Género, un destacado aporte especialmente en los aspectos conceptuales y desde un argumento que centra el análisis en las implicaciones espaciales de la relación entre producción y reproducción. A través de estas tendencias radicales en Geografía, llega a nuestra ciencia el materialismo histórico acompañado a su vez de la influencia del feminismo socialista, muy extendido en los movimientos de liberación femenina anglosajona en la década del ' 70.

En los años '80, el abordaje de nuevos temas relacionados con el fuerte crecimiento de la incorporación de la mujer en los mercados laborales, especialmente en los países occidentales, trajo consigo la necesidad de redefinir conceptos básicos como trabajo, población activa, sectores informales/formales, así como la consolidación de líneas de investigación que abordaran la participación laboral femenina y sus implicancias territoriales.

Para la década de los '90 la perspectiva de género en la ciencia geográfica ya se había afianzado y uno de sus indicios había sido el reconocimiento institucional en el seno del mundo académico, especialmente occidental: por un lado, la presencia de una geógrafa feminista en la presidencia de la Asociación de Geógrafos Americanos (9) y por otro, la consolidación de una comisión específica en los congresos de la Unión Geográfica Internacional (UGI) actúan como referencia de ese reconocimiento. Por otra parte, los avances que se produjeron en los planteamientos de género en la Geografía Humana y los resultados de investigaciones reflejados en sendas publicaciones que tratan de hacer visible a la mujer en toda su diversidad, han logrado afianzar esta perspectiva en nuestra disciplina.

\section{La perspectiva de género en la enseñanza e investigación geográfica en nuestro medio}

Como bien lo expresan Sabaté Martinez et al. (1995:47), los avances de una disciplina científica responden necesariamente a un contexto externo a la propia ciencia, como los grandes problemas sociales globales y los debates públicos que generan, hasta los cambios en las construcciones generales del pensamiento filosófico y teórico que operan en el mundo científico. En cuanto al contexto interno, es decir lo que sucede al interior de la ciencia, resulta tan interesante como aquel; en ella la "microsociedad" toma en cuenta lo que sucede en ese mundo exterior y lo traduce en la selección de los temas de investigación, en la propuesta de marcos teóricos y enfoque para su análisis. Es necesaria esta consideración porque ello explicaría el trasfondo de una escasa o nula incorporación de la perspectiva de género en los contenidos curriculares de la Geografía que se enseñan actualmente en nuestra Facultad.

\section{El contexto nacional: la situación en la Argentina de los '90}

Cuando en la década de los '90 se lleva adelante el proceso de cambio curricular en nuestra Facultad de Humanidades en el contexto de la Reforma Educativa en la Argentina, los debates sobre las aportaciones teóricas del feminismo y la incorporación de la perspectiva de género en las ciencias sociales en nuestro país aún eran incipientes (10). Señalan Veleda Da Silva y Lan (2007:111) que el enfoque de género dentro del análisis geográfico en Argentina [...] comenzó a manifestarse a fines de la década de los ochenta, pero es a mediados de los noventa cuando se inicia su definición. Hasta

Publicado en formato digital: Mgtr. Vilma Lilian Falcón. LA PERSPECTIVA DE GÉNERO EN LA CARRERA DE GEOGRAFIA DE LA UNNE. UN ESBOZO DE COMPARACIÓN CON LA INCORPORACIÓN DEL GÉNERO A LA CIENCIA GEOGRAFICA. Revista Geográfica Digital. IGUNNE. Facultad de Humanidades. UNNE. Año 10. № 19. Enero - Junio 2013. ISSN 1668-5180 Resistencia, Chaco. En:

http://hum.unne.edu.ar/revistas/geoweb/default.htm 
Revista Geográfica Digital. IGUNNE. Facultad de Humanidades. UNNE. Año 10. № 19. Enero - Junio 2013. ISSN 1668-5180 Resistencia, Chaco

entonces "la geografía estaba muy ocupada en los análisis espaciales ignorando sistemáticamente la variable de género como elemento de diferenciación dentro de la sociedad". (Colombara, 1992).

Lo que intento señalar es que la atmósfera científica reinante en el ámbito académico geográfico de nuestro país se constituye en un elemento que resulta necesario tener en cuenta al pretender analizar la Geografía enseñada en nuestro medio. Hacia fines de la década del ' 90 , período en que se produce el proceso de cambio curricular en nuestro plan de estudio, la Geografía local se encontraba todavía fuertemente ligada a los planteamientos teóricos y metodológicos provenientes del neopositivismo (11), aunque en la reflexión crítica de algunos docentes del Departamento comenzaban a perfilarse los puntales que venía imponiendo el paradigma radical (12) que estaba representado fundamentalmente por la teoría crítica, que en su transferencia a la ciencia geográfica derivaría en la denominada Geografía Crítica. Es así que, en las propuestas realizadas en el marco del cambio curricular, algunas cátedras proponían desarrollar temas que tomaran en cuenta aspectos de relevancia social como la pobreza, las desigualdades sociales y económicas y/o ambientales en los espacios locales, regionales y mundiales.

Se debe tener en cuenta que, a nivel local, las diferencias de género y sus implicancias como problema social aún no estaba instalado como tema de discusiones y reflexiones en el ámbito académico; consecuentemente los docentes e investigadores no contaban con el marco propiciatorio de debates teóricos que llevaran a reconsiderar enfoques y a redefiniciones conceptuales como para que se traduzcan en el diseño de los contenidos curriculares.

\section{Breve revisión del Plan de Estudio del Profesorado y Licenciatura en Geografía: del Plan de Estudio 1983 al Plan 2000}

En el proceso de desarrollo del Profesorado y Licenciatura en Geografía de la Facultad de Humanidades hubo siete planes de estudio. Los últimos dos (Plan 1983 y Plan 2000) son los que tomo en cuenta para sostener la observación que planteo en este trabajo, sumado a la experiencia de haber cursado el profesorado en Geografía en la década de los '80 y a mi desempeño como docente en el plantel del Departamento de Geografía en los años posteriores.

Teniendo en cuenta lo expresado y analizando los dos planes de estudio se pueden advertir algunas particularidades:

\section{Estructuras idénticas que mantienen una visión tradicional de la Ciencia Geográfica}

Tanto el Plan 1983 como el Plan 2000 conservan una estructura idéntica en el sentido que se mantiene la línea tradicional de división por niveles en los que se presenta una Geografía dividida en compartimentos: General, Física y Humana. Esta estructura (13) plantea en sus primeros ciclos el estudio del escenario terrestre como morada de la Sociedad, ésta última estudiada en los ciclos intermedios, para llegar en los últimos niveles a la explicación de la organización del espacio a través de la síntesis regional. En general esta forma de organización responde a una concepción de corte posibilista (14) y Vidaliana (15) y es la que va a dar lugar a algunos cuestionamientos por parte de algunos docentes e investigadores del Departamento como lo describiré más adelante.

En el diseño curricular vigente (Plan 2000), se advierte que en los dos primeros Niveles prevalecen las llamadas cátedras instrumentales y las generales del Área Física (16), constituyendo en conjunto el $45 \%$ del total de materias del plan de estudio. Es recién a partir del Tercer Ciclo que se incorporan cátedras que abordan temas referidos a la Geografía Humana (17) ó de los Espacios Mundiales (18). Sobre las 31 materias que se dictan actualmente, éstas últimas constituyen en conjunto un $32 \%$ del plan de estudio.

Interesa tener en cuenta este porcentaje porque de alguna manera estas áreas disciplinares podrían constituirse en el marco a través del cual se planteen nuevos temas y problemáticas de orden social desde ópticas diferentes a las tradicionales, es decir, con los elementos que proporcionan otras perspectivas epistemológicas conforme se continúe avanzando en la reflexión

Publicado en formato digital: Mgtr. Vilma Lilian Falcón. LA PERSPECTIVA DE GÉNERO EN LA CARRERA DE GEOGRAFIA DE LA UNNE. UN ESBOZO DE COMPARACIÓN CON LA INCORPORACIÓN DEL GÉNERO A LA CIENCIA GEOGRAFICA. Revista Geográfica Digital. IGUNNE. Facultad de Humanidades. UNNE. Año 10. № 19. Enero - Junio 2013. ISSN 1668-5180 Resistencia, Chaco. En:

http://hum.unne.edu.ar/revistas/geoweb/default.htm 
Revista Geográfica Digital. IGUNNE. Facultad de Humanidades. UNNE. Año 10. № 19. Enero - Junio 2013. ISSN 1668-5180 Resistencia, Chaco

teórica y metodológica de una ciencia geográfica en evolución, situación que en los últimos años ha comenzado a contemplarse en forma incipiente.

\section{Permanencia de la concepción regionalista en el estudio de los Espacios Geográficos}

Con el cambio curricular, la propuesta del Plan 2000 llega a demostrar una cierta reflexión teórico-epistemológica de la ciencia geográfica que se venía enseñando, especialmente en lo referente al enfoque regional. La discusión tenía como punto de controversia la consideración del epíteto "regional" en la denominación de las asignaturas que se ocupaban del estudio de los espacios mundiales y subespacios nacionales. Se planteaba que el término regional circunscribía a una cátedra a una única manera de abordar el estudio del Espacio Geográfico: a través del método el regional. Se sostenía que desde esta concepción no se daba lugar a otros campos de investigación o temas de análisis que estuvieran más asociados a los problemas actuales, especialmente los referidos a las cuestiones sociales. El resultado principal fue el cambio de denominación de algunas cátedras, eliminándose el epíteto "regional"; así por ejemplo, la cátedra Geografía Regional de Argentina pasa a denominarse Geografía Argentina; lo mismo sucedió con el resto de cátedras que abordan los grandes espacios regionales y mundiales. Aún así, un hecho que marca la fuerte influencia de la geografía regionalista se da en que prácticamente en todas estas cátedras actualmente se continúa abordando -especialmente como propuestas de los trabajos prácticos- el estudio de los espacios a través del método regional.

Esta observación no tiene la intención de desmerecer este método propio de la Geografía, al cual particularmente adhiero en el marco de su renovación (19); lo que intento es presentar un punto de reflexión sobre la conveniencia y necesidad de ampliar las posibilidades de análisis de los estudios geográficos con otras metodologías o propuestas renovadas, acorde con los avances metodológicos de la ciencia y de los problemas que presenta la humanidad en el mundo actual. En ese sentido, es alentador advertir algunos avances en los contenidos de los programas al proponer abordar temas actuales como los efectos de la globalización, las diferencias sociales, la pobreza, los problemas ambientales, la calidad de vida de la población, las diferencias de género, entre otros, como problemas diferenciales en los distintos espacios geográficos.

\section{Permanencia del pensamiento positivista y prevalencia del método cuantitativo en la enseñanza y la investigación}

Quienes nos formamos en la década de los '80 vivimos una fuerte influencia del pensamiento positivista; esa influencia se transmitió no solo en la enseñanza sino también en la investigación geográfica: la objetividad de la ciencia era subrayada y ello nos hacía suponer que cualquier esbozo de subjetividad no pertenecía al ámbito científico; por otro lado se insistía en el carácter neutro de los procedimientos de investigación y en la destacada relevancia de los métodos y técnicas cuantitativos. La descripción de los hechos o fenómenos geográficos de tipo social estaba centrada en datos estadísticos -censales o de encuestas- y ello era común tanto en las aplicaciones prácticas de las cátedras como en las propuestas de temas de investigación. Neutralidad, técnica y objetividad eran aspectos fuertemente resaltados a la hora de encarar cualquier análisis que se realizara en el marco de la Geografía Humana (20). El cambio curricular -1983 a 2000- ocurrió sin mayores cambios en ese sentido, por lo tanto las propuestas académicas continuaron con las mismas técnicas de análisis, reforzadas por el auge que venía teniendo el desarrollo de la informática y las posibilidades que ésta ofrecía para el tratamiento digital de los datos geográficos. Como se sabe, las estrategias cuantitativas constituyen un valioso instrumento para el análisis del complejo espacial pero no resulta la más adecuada para llevar adelante líneas de investigación más cercanas al Sujeto, particularidad que pueden ofrecer las estrategias cualitativas (21). Algunas autoras señalan que lo cuantitativo adscribe al modelo positivista de la ciencia y con sus características de objetividad y neutralidad resulta frecuentemente asociada a cualidades que se asumen como masculinas.

Publicado en formato digital: Mgtr. Vilma Lilian Falcón. LA PERSPECTIVA DE GÉNERO EN LA CARRERA DE GEOGRAFIA DE LA UNNE. UN ESBOZO DE COMPARACIÓN CON LA INCORPORACIÓN DEL GÉNERO A LA CIENCIA GEOGRAFICA. Revista Geográfica Digital. IGUNNE. Facultad de Humanidades. UNNE. Año 10. № 19. Enero - Junio 2013. ISSN 1668-5180 Resistencia, Chaco. En:

http://hum.unne.edu.ar/revistas/geoweb/default.htm 
Revista Geográfica Digital. IGUNNE. Facultad de Humanidades. UNNE. Año 10. № 19. Enero - Junio 2013. ISSN 1668-5180 Resistencia, Chaco

En este punto voy a insistir en la idea de que sin Teoría no se tiene el marco adecuado que permita reflexionar y posicionarse críticamente frente a la vida y a las realidades que el mundo nos presenta y creo ésta fue la gran ausencia que se tradujo en nuestros Planes de Estudio. Entiendo que al no haberse contemplado temas relativos a la epistemología y la influencia filosófica en el desarrollo de la Geografía, necesarios para que el alumno-investigador defina el modelo de ciencia al cual adscribe, se brindó una sola perspectiva teórica y metodológica a través de la cual se proponía observar la realidad y los problemas de orden geográfico.

De todas maneras, con la asistencia a jornadas y congresos, tanto los estudiantes como docentes de nuestra Facultad tuvimos acceso a otras realidades del país y, a través del contacto con colegas de otras universidades, se tomó conciencia de esa carencia en nuestro plan de estudio. El resultado de ese reconocimiento se tradujo posteriormente en algunos de los cambios que se destacan en el siguiente punto.

\section{Algunos aportes significativos en el Plan 2000}

Uno de los resultados más significativos fue la incorporación del estudio de la epistemología de la Ciencia Geográfica, tema que el Plan '83 no consideraba por lo que el alumno no contaba con los elementos suficientes para comprender la evolución de su propia ciencia. El resultado se tradujo no solo en la extensión del nombre de la cátedra (22) que pasó a denominarse Introducción a la Geografía, desarrollo epistemológico, sino también en la incorporación de unidades que abordan temas como: la evolución histórica del Pensamiento Geográfico (Unidad 4); la Geografía Científica (Unidad 5); la influencia Filosófica en la Geografía (Unidad 6) y la Geografía en la segunda mitad del Siglo XX (Unidad 7) (23). Destaco la relevancia de estas unidades temáticas porque brindan al alumno las pautas referenciales para comenzar a percibir e interpretar la realidad geográfica desde diferentes ópticas, brindándoles los marcos conceptuales y metodológicos de cada perspectiva teórica, condición necesaria si recordamos, como nos propone Celia Amorós (24), que la raíz etimológica de Teoría en griego significa "ver", lo que supone una forma de mirar, de observar e interpretar una determinada realidad.

Otro de los aportes significativos fue la incorporación de una nueva cátedra: "Geografía y Sociedad", la que plantea entre sus objetivos: "extender el campo de estudio de la Geografía de la población incorporando el punto de vista de las conductas sociales, es decir de la vida social de las comunidades". Resultan destacables algunos de los temas que se abordan a través de esta cátedra (25) porque constituyen espacios de reflexión para el alumno y un ámbito propicio para lograr una reconsideración y resignificación de conceptos, categorías y escalas de análisis, revisión de los principios teóricos y, a partir de ello, reconocer otros métodos alternativos de investigación.

Otro aporte concreto es el que se realiza a través de la cátedra Geografía de la Población (Área Humana) al desarrollar entre sus contenidos una unidad referida al Concepto de Género y Perspectivas en la que se abordan temas como el género en la agenda internacional, género y desarrollo, así como otras cuestiones que contribuyen a explicar las diferentes limitaciones, necesidades y oportunidades de hombres y mujeres en las distintas sociedades (26).

Por último, en otras cátedras como Geografía Económica y Política General y Geografía de África y Oceanía se han ido incorporando progresivamente espacios temáticos que favorecen la reflexión y el análisis desde un enfoque de género y, en el ámbito de la investigación, se han comenzado a generar algunas propuestas que destacan el protagonismo de las mujeres en contextos de pobreza, en el ámbito laboral y en el desempeño político.

Es cierto que la incorporación de la teoría de género requiere mucho más que temas novedosos en la docencia o en la investigación. Es ese sentido, Marcela Lagarde señala que "la perspectiva de género tiene como uno de sus fines contribuir a la construcción subjetiva y social de una nueva configuración a partir de la resignificación de la historia, la sociedad, la cultura y la política desde las mujeres y con las mujeres".(Lagarde,M. 1996:1). Desde la Geografía en general y en particular de la ciencia que se enseña e investiga en nuestra Facultad es necesaria una

Publicado en formato digital: Mgtr. Vilma Lilian Falcón. LA PERSPECTIVA DE GÉNERO EN LA CARRERA DE GEOGRAFIA DE LA UNNE. UN ESBOZO DE COMPARACIÓN CON LA INCORPORACIÓN DEL GÉNERO A LA CIENCIA GEOGRAFICA. Revista Geográfica Digital. IGUNNE. Facultad de Humanidades. UNNE. Año 10. № 19. Enero - Junio 2013. ISSN 1668-5180 Resistencia, Chaco. En:

http://hum.unne.edu.ar/revistas/geoweb/default.htm 
Revista Geográfica Digital. IGUNNE. Facultad de Humanidades. UNNE. Año 10. № 19. Enero - Junio 2013. ISSN 1668-5180 Resistencia, Chaco

resignificación de conceptos, categorías de análisis y definiciones normalmente utilizadas; de esa manera el género comenzará a ocupar el nivel de categoría explicativa que por el momento se encuentra en fases iniciales de consideración.

\section{El contexto institucional: un escenario promisorio}

Un apartado especial merece el contexto institucional que actualmente ofrece la Facultad de Humanidades de la Universidad Nacional del Nordeste en relación a esta temática y ello se debe a que en el año 2012 se formalizó un proyecto que surgió merced al trabajo integrado de docentes e investigadores de algunos Departamentos de la Unidad Académica creándose el Centro Interdisciplinario de Estudios de Género (CIDEG).

Este Centro pretende enfocarse al estudio y análisis de distintos aspectos relacionados a la perspectiva de género a través de una fuerte actividad de extensión, investigación y docencia, pero al mismo tiempo pretende establecer un fuerte vínculo con entidades y organizaciones de la región interesadas en el abordaje de problemáticas vinculadas a la cuestión de género.

El objetivo del CIDEG es lograr un abordaje integral de la perspectiva de género, que no se reduzca sólo a la problemática social sino que también busque identificar el concepto de género en los discursos, leyes, actas, en la historia regional, en obras literarias, y en otras tantas formas en las que la sociedad exponga su visión o visiones respecto a la idea de género.

En el marco de reflexión que venimos realizando en este artículo nos interesa destacar dos cuestiones que se proyecta promover desde el Centro: fomentar investigaciones, estudios, conferencias, talleres, encuentros intercátedras y otras actividades que permitan debatir y difundir el tema y propiciar la inclusión de la temática en los programas curriculares de las asignaturas así como la realización de actividades específicas relacionadas al género. En este sentido, entendemos que institucionalmente se ha comenzado a trabajar para generar ese espacio que fomente la discusión teórica de temas hasta ahora ignorados o insuficientemente explicados desde nuestra ciencias y que reflejen ese "salto conceptual" al que Marta Lamas nos invita a realizar cuando nos señala que la teoría no es un lujo sino una necesidad " no solo para facilitar el indispensable cambio de paradigmas sobre la condición humana, sino para frenar las prácticas discriminatorias que traducen diferencia por desigualdad". (Lamas, M.,2004:2).

\section{A modo de Conclusión}

Hemos advertido, a través de este breve repaso, que la incorporación de la perspectiva de género a la ciencia geográfica fue dándose en forma paulatina pero sostenida, ello fue así tanto en el marco de evolución de la Geografía en el mundo como también de la Geografía en nuestro país.

Al analizar la situación de la Geografía que se enseña e investiga en nuestra Facultad, creo que el camino ha comenzado a transitarse, muy lentamente y con un considerable retraso respecto de otras universidades del mundo y del país, situación que podríamos considerar como el contexto externo en la justificación de porqué la perspectiva de género no fue considerada en nuestros planes de estudios vigentes. Por otra parte, aunque no desligada totalmente de la consideración anterior, hemos destacado el fuerte lazo que mantuvo la "microsociedad" académica de geógrafos locales con las líneas del Pensamiento Geográfico Neopositivista, marco no del todo adecuado para poner en práctica los principios teóricos y prácticos que requiere la perspectiva de género. Ello explicaría el hecho de que en los momentos actuales solo una ínfima parte de los programas curriculares del profesorado y licenciatura en Geografía exponen tímidamente temas relacionados con las "cuestiones de género"; por lo que podríamos parafrasear con Marcela Lagarde (1996:13) señalando que en nuestros planes de estudio aún permanece "una concepción androcéntrica de la humanidad, dejando fuera a la mitad del género humano".

$\mathrm{Si}$, como lo señalan las autoras en cuya obra me he basado para lograr esta comparación, la propuesta de la Geografía del Género es lograr un replanteo de las formas tradicionales de practicar nuestra disciplina, entiendo que el reconocimiento de esa necesidad es ya un avance importante para

Publicado en formato digital: Mgtr. Vilma Lilian Falcón. LA PERSPECTIVA DE GÉNERO EN LA CARRERA DE GEOGRAFIA DE LA UNNE. UN ESBOZO DE COMPARACIÓN CON LA INCORPORACIÓN DEL GÉNERO A LA CIENCIA GEOGRAFICA. Revista Geográfica Digital. IGUNNE. Facultad de Humanidades. UNNE. Año 10. № 19. Enero - Junio 2013. ISSN 1668-5180 Resistencia, Chaco. En:

http://hum.unne.edu.ar/revistas/geoweb/default.htm 
Revista Geográfica Digital. IGUNNE. Facultad de Humanidades. UNNE. Año 10. № 19. Enero - Junio 2013.

ISSN 1668-5180 Resistencia, Chaco

comenzar la búsqueda de reinterpretación de una Geografía que demuestre que el espacio no es neutro desde el punto de vista del género. Esto no es más que comprender que "las implicaciones de género en el estudio de la Geografía son por lo menos tan importantes como las implicaciones de cualquier otro factor social o económico que transforme la sociedad y el espacio" (Women and Geography Study Group, 1984:21) (27).

Este es un desafío al cual algunos docentes e investigadores de nuestro Departamento en particular y de nuestra unidad académica en general ha elegido enfrentarse, con la idea esperanzadora de contribuir, desde la Geografía y demás Ciencias Sociales, a dar visibilidad a la mitad del género humano.

\section{Notas}

(1) Muchas veces el término género se convierte en un eufemismo que engloba varias cosas: mujeres, relaciones entre los sexos y feminismo y genera cierta ambigüedad que, como lo señala Marta Lamas (2004), favorece un ocultamiento con el cual se evita precisar que hay discriminación u opresión".

(2) Citado en De Miguel Alvarez, Ana (2000:1)

(3) Sabaté Martinez, A.; Rodriguez Moya, J. y Diaz Muñoz, M.A. (1995)

(4) Se tiene en cuenta este proceso de cambio curricular operado en la década de los '90 dado que en esa época los debates sobre las aportaciones teóricas del feminismo y la incorporación de la perspectiva de género en las ciencias sociales en nuestro país comenzaban a darse en forma incipiente.

(5) Resulta interesante el análisis que realiza María Luisa Femenías (2005-2006) en su cartografía preliminar del feminismo latinoamericano al ilustrar claramente el problema del origen de este movimiento en América Latina.

(6) Marta Lamas (2004) realiza un interesante aporte acerca del desarrollo e incorporación del concepto de género en la Antropología.

(7) La revisión acerca de las aportaciones de las distintas corrientes fue realizado en base al Capítulo 2 de Sabaté Martinez, A.; Rodriguez Moya, J. y Diaz Muñoz, M.A. (1995- págs. 24-55)

(8) Citada en: Sabaté Martinez, A.; Rodriguez Moya, J. y Diaz Muñoz, M.A. Cap.2 (1995:30)

(9) Se trata de la géografa feminista Susan Hanson quien asumió la presidencia de Association of American Geographers en el año 1992.

(10) Los aportes que analizan el proceso que siguió la incorporación de género a las ciencias sociales, especialmente a la Historia y la Geografía, coinciden en señalar que si bien los estudios sobre mujeres comienzan a sobresalir entre los '60 y '80 es después del advenimiento de la democracia en 1983 cuando cobran vigor, especialmente las publicaciones que desde la historia daban cuenta del rol de las mujeres en el tiempo (Cfr. Valobra, A.M. 2005:2). Por su parte, el enfoque de género dentro del análisis geográfico en Argentina es más reciente, (Veleda Da Silva y Lan, 2007:111) y ha cobrado relevancia en los '90 como resultado de los debates suscitados en eventos académicos como jornadas y congresos y el consiguiente incremento de producción acerca de esta temática, situación que también es observable en la Historia.

(11) Señala Baxendale, C. (2007:31) que con la aparición de las corrientes denominadas neopositivistas se origina "una vuelta a la aplicación de teorías físicas al campo de las ciencias humanas y nuevamente la búsqueda de leyes generales, ahora de carácter probabilístico, mediante, ya no el método netamente inductivo, sino principalmente hipotético-deductivo al insistirse en la necesidad de teorías previas." El análisis espacial está orientado por los métodos cuantitativos, las técnicas y la construccion de modelos.

(12) El paradigma radical plantea, precisamente, una posición radicalmente opuesta al paradigma cuantitativo (predominante en la Geografía de mediados de la década del ' 50 hasta principios de los '70), planteando un interés por las diferencias que el desarrollo económico mostraba a escala planetaria (impuestas por el modo de producción capitalista), las relaciones de dependencia y

Publicado en formato digital: Mgtr. Vilma Lilian Falcón. LA PERSPECTIVA DE GÉNERO EN LA CARRERA DE GEOGRAFIA DE LA UNNE. UN ESBOZO DE COMPARACIÓN CON LA INCORPORACIÓN DEL GÉNERO A LA CIENCIA GEOGRAFICA. Revista Geográfica Digital. IGUNNE. Facultad de Humanidades. UNNE. Año 10. № 19. Enero - Junio 2013. ISSN 1668-5180 Resistencia, Chaco. En:

http://hum.unne.edu.ar/revistas/geoweb/default.htm 
Revista Geográfica Digital. IGUNNE. Facultad de Humanidades. UNNE. Año 10. № 19. Enero - Junio 2013.

ISSN 1668-5180 Resistencia, Chaco

explotación involucradas en estas diferencias, y sus consecuencias en las condiciones de vida. "La Geografía Cuantitativa es criticada por su falta de compromiso y porque sus estudios, de poca relevancia social, sirven para hacer perpetuar el sistema y al mismo tiempo mantener el status quo." (Cfr. Buzai, G. (2006:36-37)

(13) En el Plan de estudio 2000 se explica dicha estructura: EL primer ciclo o ciclo instrumental. Su duración es de 1 año. Introduce a los alumnos en los campos disciplinares, desarrollando los conocimientos y técnicas básicas para el aprendizaje de los contenidos y su aplicación en los ciclos siguientes. El sequndo ciclo o de formación básica. Su duración es de 3 años. Desarrollo de los fundamentos teóricos y metodológicos de la ciencia geográfica, permitiendo aplicar las interrelaciones hombre-medio, sociedad - ambiente, sus respuestas y resultados históricos y actuales sobre la organización del espacio. El tercer ciclo o ciclo de formación profesional. Duración de un año Profundización y aplicación de los conocimientos teóricos prácticos y metodológicos de la ciencia geográfica en la docencia e investigación.

(14) El posibilismo surge en la primera mitad siglo XX. Tiene como base filosófica el idealismo y el historicismo. Su postulado básico es que el medio natural ofrece unas posibilidades de desarrollo, que las sociedades aprovechan o no, en función de su nivel técnico y grado de organización social. Su núcleo está en Francia y el "padre del posibilismo" es Paul Vidal de la Blache, mientras que en Alemania el más destacado es Alfred Hettner y en EEUU Carl Ortwin Sauer.

(15) Paul Vidal de La Blache (1845-1918) es considerado uno de los geógrafos más importantes de la segunda mitad del siglo XIX. Para Vidal de La Blache, el objeto de la geografía era la relación hombre-naturaleza, desde la perspectiva del paisaje, del estudio de la región. Al hombre lo considera un ser activo, que sufre la influencia del medio, actuando sobre éste y transformándolo. Bajo esta perspectiva la naturaleza se considera como un conjunto de posibilidades para la acción del hombre. Revolucionó la Geografía dándole un objeto de estudio bien diferenciado y exclusivo, de tal manera que no se podía confundir con ninguna otra ciencia: la región.

(16) Con excepción de la cátedra "Introducción a la Geografía, desarrollo epistemológico".

(17) Geografía de la Población; Geografía Económica y Política General; Geografía Urbana y Agraria; Geografía y Sociedad; Historia Económica y Social Argentina.

(18) Geografía Argentina; Geografía de América; Geografía de Europa y Asia; Geografía de África y Oceanía y Seminario del Nordeste Argentino.

(19) En las últimas décadas surgió la llamada Nueva Geografía Regional que revaloriza la diversidad y las diferencias espaciales como objeto de investigación geográfica. Estas diferencias constituyen la respuesta individualizada de cada "localidad", con su propia población e identidad cultural/histórica, a los procesos generales y propone nuevos métodos y técnicas -algunas provenientes de otras ciencias sociales- para contribuir a explicar porqué existen espacios diferentes pero enfrentados a problemas comunes.

(20) Janice Monk, geógrafa feminista de origen australiano y formada en el mundo anglosajón, relata en su biografía una situación similar en sus inicios como investigadora: "Hacer un trabajo de prestigio en geografía en aquel momento consistía en construir modelos matemáticos, buscar leyes universales y trabajar con datos de censos o similares, y sobre todo en temas urbanos..." (Monk, Janice, 2007:23)

(21) No se desconoce el aporte que las técnicas cuantitativas pueden ofrecer a las líneas de investigación con perspectiva de género, dado que resultan muy útiles en las fases descriptivas del fenómeno. Por otra parte, es necesario considerar que dentro del Positivismo también se puede realizar una investigación válida desde la perspectiva de género, siempre y cuando exista la necesaria coherencia entre filosofía, teoría, método y técnicas de análisis.

(22) En el Plan 1983 figura como Introducción a la Geografía.

(23) Se puede acceder al programa completo a través del siguiente sitio:

http://hum.unne.edu.ar/academica/departamentos/dptogeog/catedras.htm

(24) Citada en Ana de Miguel Alvarez (2000:1)

(25) Esta cátedra, introducida en el Plan 2000, contempla entre sus contenidos mínimos temas como: Fundamentos de las principales corrientes teóricas del pensamiento social y su influencia en el

Publicado en formato digital: Mgtr. Vilma Lilian Falcón. LA PERSPECTIVA DE GÉNERO EN LA CARRERA DE GEOGRAFIA DE LA UNNE. UN ESBOZO DE COMPARACIÓN CON LA INCORPORACIÓN DEL GÉNERO A LA CIENCIA GEOGRAFICA. Revista Geográfica Digital. IGUNNE. Facultad de Humanidades. UNNE. Año 10. № 19. Enero - Junio 2013. ISSN 1668-5180 Resistencia, Chaco. En:

http://hum.unne.edu.ar/revistas/geoweb/default.htm 
Revista Geográfica Digital. IGUNNE. Facultad de Humanidades. UNNE. Año 10. № 19. Enero - Junio 2013.

ISSN 1668-5180 Resistencia, Chaco

pensamiento geográfico; las principales cuestiones de análisis de la vida social de la población; Modos de segregación...;La familia y el hogar como unidades de toma de decisión; Movimientos sociales, entre otros.

(26) El tratamiento de estos temas figura en el libro "Geografía de la Población. La composición de la población" cuya autora es Ana María Foschiatti, Profesora Titular de la cátedra (Foschiatti, A.M. 2009:78-108)

(27) Citado en Sabaté Martinez, et al (1995:18)

\section{Bibliografía}

- BAXENDALE, Claudia (2007). Región y Estudios regionales. Consideraciones desde los diferentes enfoques de la Geografía. En: Fronteras, № 6 - Año 6, Octubre de 2007.

- BUZAI, Gustavo y BAXENDALE, Claudia (2006) Análisis Socioespacial con Sistemas de Información Geográfica. Grupo de Ecología del Paisaje y Medio Ambiente. Universidad de Buenos Aires- Lugar Editorial.

- COLOMBARA, Mónica (1992). Espacio y mujer. Una contribución a la geografía del género. Boletín de Estudios Geográficos. UNCuyo, Mendoza, vol. 25, nº 89, p. 25-33.

- DE LAURETIS, Teresa (1993). Sujetos excentricos: la Teoría Feminista y la Conciencia Historica. Versión tomada del libro: De mujer a género, Teoría, interpretación y práctica feministas en las ciencias sociales; María C. Cangiamo y Lindsay DuBois, comp.; Centro Editor de América Latina; Buenos Aires; 1993; 161pp; (págs. 73-113).

- DE MIGUEL ÁlVAREZ, Ana (2000). Movimiento feminista y redefinición de la realidad. Mujeres en Red. Universidad de la Coruña.

- ESTEBANEZ, José (1982) Tendencias y problemática actual de la Geografía. Cuadernos de Estudio 1. Serie Geografía. Editorial Cincel, Madrid. España.

- FEMENIAS, María Luisa (2005-2006). El Feminismo Iberoamericano: cartografía preliminar. Publicado en Pasajes (Universidad de Valencia). № 19 (2005-2006)

- FOSCHIATTI, A.M. (2009). Geografía de la Población. La composición de la población. Instituto de Geografía. Facultad de Humanidades. UNNE, Resistencia, Chaco.

- LAGARDE, Marcela (1996). "El género", fragmento literal: 'La perspectiva de género', en Género y feminismo. Desarrollo humano y democracia, Ed. horas y HORAS, España.

- LAMAS, Marta (2004). Género: algunas precisiones conceptuales y teóricas. Conferencia Magistral presentada en el XIII Coloquio Anual de Estudios de Género, en la Ciudad de México, el 17 de noviembre del 2004.

- MONK, Janice (2007). Generizando la Geografía; personas lugares e ideas. Document Anàlisi Geografico. 49. Universitat Autonoma de Barcelona. España

- RIUDOR, Lluis (1988). La Geografía Regional del mundo y sus planteamientos metodológicos recientes. Documents D'Analisi Geografica. № 13. pags. 81-89. Departament de Geografia. Universitat Autónoma de Barcelona.España.

- SABATE mARTINEZ, Ana, RODRIGUEZ MOYA, Juana y DIAZ MUÑOZ, M. los Angeles. (1995). Mujeres, Espacio y Sociedad. Hacia una Geografía del Género. Editorial SINTESIS, S.A. Madrid, España.

- VELEDA DA SILVA, Susana M. y LAN, Diana (2007). Estudios de geografía del género en América Latina: un estado de la cuestión a partir de los casos de Brasil y Argentina. Doc. Anàl. Geogr. 49, $2007 \quad 99-118 . \quad$ Disponible en: http://ddd.uab.cat/pub/dag/02121573n49p99.pdf. Consultado [6 de febrero de 2012 ] 